\title{
The potential role of Th17 lymphocytes in patients with psoriasis*
}

\author{
Mahnaz Mansouri ${ }^{1}$ \\ Abbas Ali Raze ${ }^{3}$
}

\author{
Parvine Mansouri ${ }^{2}$ \\ Zohreh Jadali ${ }^{4}$
}

DOI: http:/ / dx.doi.org/10.1590/abd1806-4841.20186123

\begin{abstract}
BACKGROUND: Psoriasis is a chronic inflammatory disorder, characterized by increased keratinocyte proliferation due to abnormal differentiation of basal keratinocytes. The etiology of the disease is unclear, and according to the survey results, it is hypothesized that a combination of genetic and environmental factors prompts an abnormal immune response in patients with psoriasis. CD4+ Th cells play a multifaceted role in both immune defense and pathogenesis of certain diseases such as psoriasis. Nonetheless, the exact contribution of different subpopulations of Th cells in psoriasis is still not clear.

OвJEстіvE: The aim of present study was to determine the mRNA expression level of RORC as potential inducer of Th17 cell differentiation and expression pattern of Th17-signature cytokines (IL-17A and IL-22).

Methods: Twenty patients with psoriasis and twenty-one healthy subjects were included in the study. Peripheral blood mononuclear cells (PBMCs) were separated and expression of three genes were determined by quantitative real-time reverse transcriptase PCR (qRT-PCR). Plasma levels of IL-17 and IL-22 were also evaluated by ELISA.

RESULTS: RORC, IL-17A and IL-22 gene expression was significantly higher in patients with psoriasis compared with healthy controls $(\mathrm{P}<0.05)$. In addition, a marked increase in plasma IL-17A and IL-22 levels was observed in patient group compared to controls $(\mathrm{P}<0.001)$.

STUDY LIMITATIONS: small number of patients.
\end{abstract}

Conclusion: These data suggest that Th17 response may contribute to the pathogenesis of psoriasis.

Keywords: Autoimmunity; Cytokines; Psoriasis; T-Lymphocytes

\section{INTRODUCTION}

Psoriasis is a chronic inflammatory disorder, characterized by increased keratinocyte proliferation due to abnormal differentiation of basal keratinocytes. The etiology of the disease is unclear, and according to the survey results, it is hypothesized that a combination of genetic and environmental factors prompts an abnormal immune response in patients with psoriasis. ${ }^{1}$

Among different cellular elements of the immune system, $\mathrm{T}$ cells have received considerable attention because of their importance in disease pathogenesis. $\mathrm{T}$ lymphocytes are heterogeneous and consist of distinct populations that can be defined depending on surface markers and effector functions (such as their secreted cytokine profile and cytotoxicity or cytoprotective effects). Moreover, differentiation and function of each $\mathrm{T}$ cell subset are regulated by the activity and relative expression of specific transcription factors. These differences provide suitable tools for identification of different subsets of T cells. ${ }^{2}$

Th17-cells are special subpopulations of CD4+ T-cells that produce proinflammatory cytokines such as IL-17A and IL-22 and express lineage-specific transcription factor RORC. These cells play a key role in host defense against extracellular pathogens. However, under certain circumstances, they have also been implicated in the pathogenesis of a variety of autoimmune and inflammatory diseases including psoriasis., ${ }^{2,3}$

Augmented or unbalanced activation of T cells with shift toward Th17 responses, not only disturb appropriate immune responses but also provide suitable conditions for the development

Received on 06.06.2016.

Approved by the Advisory Board and accepted for publication on 06.12.2016.

* Work performed at the Tehran University of Medical Sciences - Tehran, Iran. Financial support: Tehran University of Medical Sciences.

Conflict of interest: None.

Department of Molecular Medicine, Pasteur Institute of Iran, Tehran, Iran.

Department of Dermatology and Laser Surgery, Skin and Stem cell Research Center, Tehran University of Medical Sciences, Tehran, Iran.

Department of Medical Biotechnology, Biotechnology Research Center (BRC), Pasteur Institute of Iran, Tehran, Iran.

Department of Immunology, School of Public Health, Tehran University of Medical Sciences. Tehran, Iran.

MAILING ADDRESS:

Zohreh Jadali

E-mail: zjadali@razi.tums.ac.ir; zjadali@yahoo.co.uk

(C2018 by Anais Brasileiros de Dermatologia 
of psoriasis. However, there is no consensus in the literature on this issue. ${ }^{4-6}$ Hence, identification of molecular mechanisms that control the specification and differentiation of CD4+ T cells into Th17 effector cells in diseased subjects will substantially improve our insight into disease pathogenesis. Therefore, the goal of this research was to (1) determine RORC, IL-17A, and IL-22 mRNA expression patterns in PBMCs of patients with psoriasis and healthy control subjects, (2) analyze the correlation between RORC and IL-17A/IL-22 to understand the potential role of this transcription factor in signaling for the differentiation of Th17 lymphocytes, and (3) to assess whether there is a difference in cytokine levels (IL-17A and IL-22) between healthy subjects and patients with psoriasis.

\section{METHODS}

\section{Sampling}

Five milliliters of peripheral blood were collected from each individual in EDTA-containing tubes and processed for PBMC preparation using Ficoll density-gradient centrifugation (Pharmacia, Uppsala, Sweden). The obtained blood plasma was also stored at $-80^{\circ} \mathrm{C}$ until analysis. The study group comprised of 20 patients with psoriasis (14 women and 6 men) with a mean age of 36.25 years (range, 17-74 years). Newly diagnosed patients who had not received previous treatment for psoriasis and medication-free subjects for at least 4 weeks of the study were included in this research project. Patients suffering from inflammatory/autoimmune disorders other than psoriasis were excluded. Twenty-one healthy subjects who had no history of psoriasis or other chronic and autoimmune diseases were enrolled in the study. They consisted of 8 men and 13 women, with a mean age of 36.86 years (range, 25-60 years). The severity of psoriasis determined by the psoriasis area and severity index (PASI) for each patient. The mean PASI of twenty participants was $7.14 \pm 7.43$ points.

Informed written consent has been completed on $100 \%$ of the cases recruited for this study. The study protocol was approved by the Ethical Committee of Tehran University of Medical Sciences and was in accordance with the principles of the Declaration of Helsinki. RNA isolation, cDNA synthesis and quantitative real-time polymerase chain reaction (qRT-PCR) were determined.

Total RNA was extracted from PBMCs using RNX-plus solution (Sinaclon BioScience Co, Tehran, Iran) according to manufacturer's instructions. RNA solution has been qualified by measuring ratio of optical density (OD) 260/280 on a Nanodrop spectrophotometer (Thermo Scientific, USA), and solutions having OD260/280 ratio below 1.6 were discarded. Each RNA sample was reverse transcribed by RevertAid First Strand cDNA Synthesis Kit (Thermo Scientific, USA) as recommended by the manufacturer. These cDNAs were used as a template and were amplified by SYBR Premix EX Taq II (Takara, Japan) on a RotorGene 6000 (Corbett Life Science, Australia) thermal cycler. The PCR was performed in a total volume of $10 \mu \mathrm{l}$ containing $5-\mu l$ SYBR Premix, $1-\mu l$ cDNA, $0.5-\mu l$ of forward and reverse primers, and 3- $\mu 1$ double distilled water. The amplification took place in a twostep PCR and thermal cycling conditions consisted of an initial denaturation step for $2 \mathrm{~min}$ at $95^{\circ} \mathrm{C}$ followed by $38-45$ cycles of $5 \mathrm{~s}$ at $95^{\circ} \mathrm{C}$ (denaturation) and final step of $20 \mathrm{~s}$ at $62^{\circ} \mathrm{C}$ for RORC, $45 \mathrm{~s}$ at $57^{\circ} \mathrm{C}$ for IL-17A, 45 s at $60^{\circ} \mathrm{C}$ for IL-22, and 20 s at $63^{\circ} \mathrm{C}$ for $\beta$-actin.
PCR primers pairs were designed by using OLIGO software (National Biosciences) and purchased from Gene fanavaran (Tehran, Iran). The specific sequences of primers used in this study are shown in table 1.

Relative quantities for all samples were normalized to their corresponding $\beta$-actin levels. The cycle threshold(Ct) method was used to determine the amplification of the genes relative to $\beta$-actin as endogenous control. The $\mathrm{Ct}$ levels are inversely proportional to the abundance of a target mRNA in the samples. Therefore, lower $\mathrm{Ct}$ levels reveal elevated amounts of mRNA, whereas higher $\mathrm{Ct}$ levels indicate decreased amounts of mRNA.

\section{Cytokines assay}

The levels of IL-17A and IL-22 were determined using an enzyme-linked immunosorbent assay method according to the manufacturer's instructions (Bender Med Systems, San Diego, California, USA).

\section{Statistical Analysis}

Statistical comparison between groups was performed by the T-tests or Pearson test, using the Statistical Package for Social Sciences (SPSS), version 22.0 (SPSS, Inc., Chicago, IL). The level of significance was set at $\mathrm{P}<0.05$. Results are expressed as means $\pm \mathrm{SD}$.

\section{RESULTS}

The expression of RORC, IL-17A and IL-22 in patients with psoriasis and healthy subjects

As shown in figure 1, RORC expression was significantly increased in patients $(6.32 \pm 1.56)$, as compared to healthy controls $(7.98 \pm 1.78)(\mathrm{P}=0.003)$. The mean expression levels of IL-17A were also significantly higher $(\mathrm{P}=0.015)$ in patients $(13.07 \pm 3.73)$ than in controls $(16.07 \pm 3.80)$ (Figure 2). Similar results were observed for mRNA expression of IL-22 in psoriatic patients (14.76 \pm 2.47$)$ in comparison with the control group $(16.77 \pm 2.05)(\mathrm{P}=0.007)$ (Figure 3$)$.

\section{Alterations in plasma inflammatory cytokines of patients with psoriasis}

The cytokines examined in the present study were detected in plasma samples of all patients and controls. Both IL-17A (P = $0.043)$ and IL-22 ( $=0.001)$ concentrations were significantly higher in psoriatic patients than in healthy controls (Figure 4).

\section{Correlation analysis}

The mRNA expression of RORC correlated positively with mRNA expression of IL-17A $(\mathrm{r}=0.465, \mathrm{P}=0.04)$ and IL-22 $(\mathrm{r}=$ $0.507, \mathrm{P}=0.02)$. Statistical analysis indicated that in patients, IL-17A

\section{TABLE 1: Primer sequences for the real-time quantitative PCR}

\begin{tabular}{ll} 
RORC & F: 5'AGGAAGTCCATGTGGGAG3' \\
& R: 5'AGCACAATCTGGTCATTCTGG3' \\
IL-17 & F: 5'CTCATTGGTGTCACTGCT3' \\
& R: 5'CGGTTGTAGTAATCTGAGG' \\
IL-22 & F: 5'TGCTGTTCCCTCAATCTG3' \\
& R: 5'TGTGCTTAGCCTGTTGCTG3' \\
\hline \multirow{2}{*}{-actin } & F: 5'AGACGCAGGATGGCATGGG' \\
& R: 5'GAGACCTTCAACACCCCAGCC3
\end{tabular}

$F$, forward primer, $R$, reverse primer 
mRNA level was significantly correlated with IL-22 mRNA level ( $\mathrm{r}$ $=0.445, \mathrm{P}=0.04)$. There was also good correlation between IL-17A and IL-22 mRNA expression and plasma IL-17A and IL-22 secretion respectively $(\mathrm{P}<0.001)$. There was no correlation between the above-mentioned parameters and severity of disease $(\mathrm{P}>0.05)$.

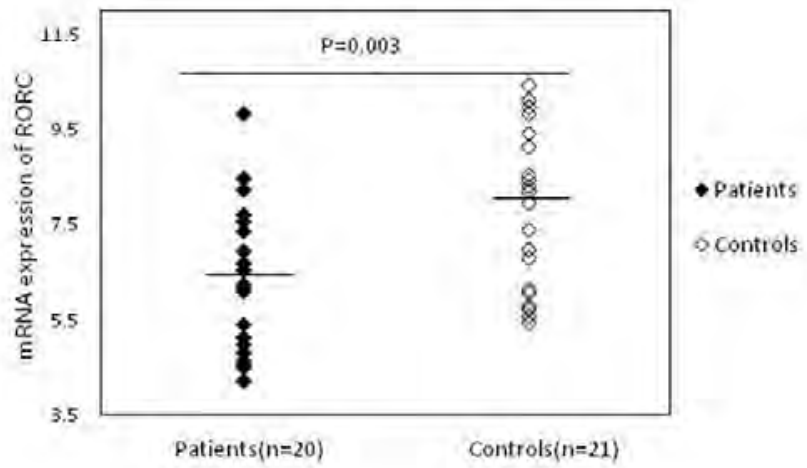

FIGURE 1: mRNA expression levels for RORC in patients and control subjects. $\beta$-acttin was used as the internal control. Please note that a higher $\Delta \mathrm{Ct}$ value corresponds to a comparably lower expression level

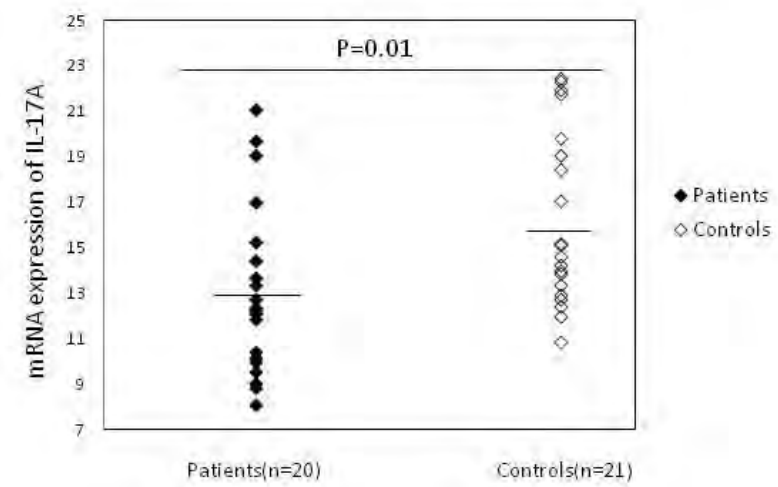

FIGURE 2: mRNA expression levels for IL-17A in patients and control subjects. $\beta$-action was used as the internal control. Please note that a higher $\Delta \mathrm{Ct}$ value corresponds to a comparably lower expression level

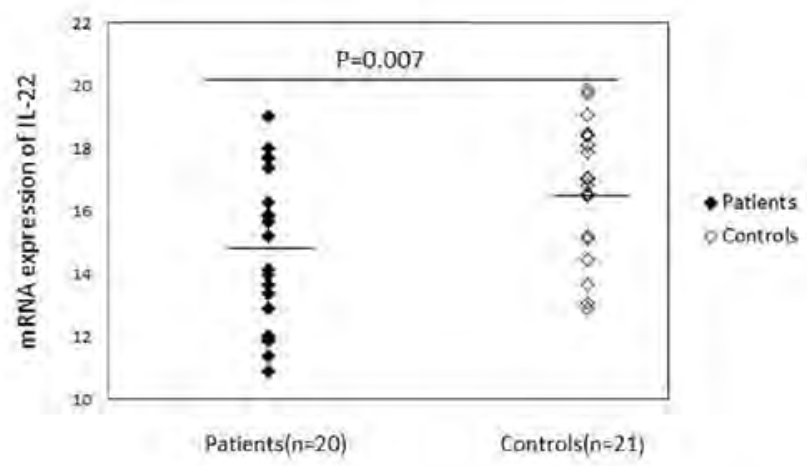

FIGURE 3: mRNA expression for IL-22 in patients and control subjects. $\beta$-action was used as the internal control. Please note that a higher $\Delta \mathrm{Ct}$ value corresponds to a comparably lower expression level

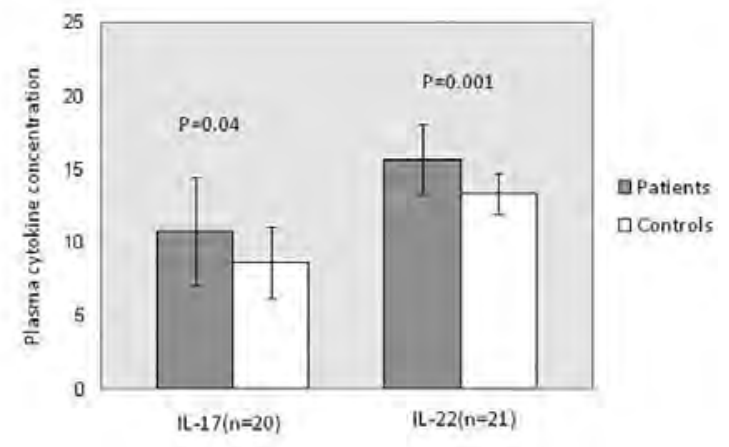

Figure 4: Plasma cytokine levels of psoriasis patients and controls

\section{DISCUSSION}

Psoriasis has been described by hyperproliferation and aberrant differentiation of keratinocytes. Although the exact mechanism(s) of psoriasis is still unknown, it is assumed that T cells play a dominant pathogenic role in the initiation and persistence of disease. Numerous lines of evidence suggest that activated T cells are the primary components in the pathogenesis of psoriasis. The implication of T cells in the process of disease development is supported by various observations such as elevated levels of activated T lymphocytes in blood and skin lesions of patients with psoriasis. ${ }^{7,8}$ Moreover, it was indicated that CD4+ T cells from psoriatic patients can transfer the same disease to experimental animals. ${ }^{9} \mathrm{CD} 4+\mathrm{T}$ cells have a critical role in adaptive immunity and belong to a diverse set of subpopulations with particular functions and molecular phenotypes. Different subsets of $\mathrm{T}$ cells originate from a non-polarized, naïve $\mathrm{T}$ cells with stem cell-like properties. Naïve $\mathrm{T}$ cells can differentiate into all different types of effector and memory cells upon $\mathrm{T}$ cell priming. For many years, psoriasis was regarded as a Th1 cell-mediated disease, however, it is now obvious that a specific IL17 and IL-22-producing CD4+ T cell subset contribute to the pathogenesis of this important skin disease. The significance of Th17 cells in psoriasis comes from clinical and experimental studies. ${ }^{2,10}$

Therefore, the first step of this survey was conducted to compare the expression pattern of Th17-specific transcription factor RORC in PBMCs of patients with psoriasis and control subjects. Our results indicated a considerable increase in RORC mRNA level in PBMCs of patients compared to healthy controls and are consistent with earlier studies showing elevated expression of RORC transcripts in peripheral blood of patients with psoriatic arthritis. ${ }^{11}$ Increased transcript level of RORC was also encountered in skin lesions of psoriatic patients when compared with uninvolved skin and healthy control skin. ${ }^{12}$ Therefore, gene expression changes of RORC mRNA may be implicated in the pathogenesis of psoriasis.

In another part of this study, the expression levels of IL-17A and IL-22 were analyzed. Moreover, the plasma levels of these cytokines were measured by ELISA. Our attention was focused on these cytokines because of their importance as a major cytokine product of Th17 cells. Furthermore, altered production of these cytokines are thought to play a critical role in pathogenic host immune responses. 
Our results indicated a major increase of IL-17A and IL-22 mRNA level in patients compared with healthy controls $(\mathrm{P}<0.05)$. A similar pattern was also observed for the protein levels of these cytokines.

Moreover, the level of RORC was significantly correlated with IL-17A $(r=0.465, \mathrm{P}=0.04)$ and IL-22 $(\mathrm{r}=0.507, \mathrm{P}=0.02)$ mRNA expression. IL-17A and IL-22 mRNA expressions were also significantly correlated with plasma IL-17A $(r=0.983 ; \mathrm{P}<0.001)$ and IL-22 $(\mathrm{r}=0.835 ; \mathrm{P}<0.001)$ secretion respectively. These data are in good agreement with earlier studies indicating increased expression of IL-17A and IL-22 transcripts in patients with psoriasis. For instance, Lowes et al. found increased expression of IL-17 and IL-22 mRNA in skin lesions of patients with psoriasis compared to non-lesional skin..$^{13}$ In another study, Roh et al. found a significant increase in expression levels of IL-17A and IL-22 in the lesions of psoriasis patients compared to healthy controls. ${ }^{14}$

Although the results of present study demonstrate significant differences between the patients and controls groups in the mean concentrations of plasma IL-17 and IL-22, there is no consensus in the literature regarding the patterns of the above-mentioned cytokines in psoriasis. For instance, Arican et al. found no significant differences in the plasma levels of IL-17 between the patient and control groups. ${ }^{5}$ Similar results were also observed concerning IL-22 levels in the plasma of patients with psoriasis, compared to those of healthy controls. ${ }^{14}$ These differences may be related to different factors, including treatment, various clinical subtypes of disease, and disease severity. ${ }^{15,16}$

A correlation analysis between psoriasis severity (PASI

\section{REFERENCES}

1. Raychaudhuri SP. A cutting edge overview: psoriatic disease. Clin Rev Allergy Immunol. 2013;44:109-13

2. Maddur MS, Miossec P, Kaveri SV, Bayry J. Th17 cells: biology, pathogenesis of autoimmune and inflammatory diseases, and therapeutic strategies. Am J Pathol. 2012;181:8-18.

3. Deng Y, Chang C, Lu Q. The Inflammatory Response in Psoriasis: a Comprehensive Review. Clin Rev Allergy Immunol. 2016:50:377-89.

4. Marinoni B, Ceribelli A, Massarotti MS, Selmi C.The Th17 axis in psoriatic disease: pathogenetic and therapeutic implications. Auto Immun Highlights. 2014;5:9-19.

5. Arican 0, Aral M, Sasmaz S, Ciragil P. Serum levels of TNF-alpha, IFN-gamma, $\mathrm{IL}-6, \mathrm{IL}-8, \mathrm{IL}-12, \mathrm{IL}-17$, and IL-18 in patients with active psoriasis and correlation with disease severity. Mediators Inflamm. 2005;2005:273-9.

6. Fitch EL, Rizzo HL, Kurtz SE, Wegmann KW, Gao W, Benson JM, et al. Inflammatory skin disease in K5. hTGF- $\beta 1$ transgenic mice is not dependent on the IL-23/Th17 inflammatory pathway. J Invest Dermatol. 2009;129:2443-50.

7. Cabrijan L, Lipozencić J, Batinac T, Peternel S, Pastar Z. The role of CD4 and CD8 lymphocytes and macrophages in psoriasis vulgaris. Acta Dermatovenerol Croat. 2009;17:162-5.

8. Kagami S, Rizzo HL, Lee JJ, Koguchi Y, Blauvelt A. Circulating Th17, Th22, and Th1 cells are increased in psoriasis. J Invest Dermatol. 2010;130:1373-83.

9. Nickoloff BJ, Wrone-Smith T. Injection of pre-psoriatic skin with CD4+ T cells induces psoriasis. Am J Pathol. 1999;155:145-58

10. Nadeem A, Al-Harbi NO, Al-Harbi MM, El-Sherbeeny AM, Ahmad SF, Siddiqui $\mathrm{N}$, et al. Imiquimod-induced psoriasis-like skin inflammation is suppressed by BET bromodomain inhibitor in mice through RORC/IL-17A pathway modulation. Pharmacol Res. 2015;99:248-57.

11. Leipe J, Grunke M, Dechant C, Reindl C, Kerzendorf U, Schulze-Koops H, et al. Role of Th17 cells in human autoimmune arthritis. Arthritis Rheum. 2010;62:2876-85.

12. Wilson NJ, Boniface K, Chan JR, McKenzie BS, Blumenschein WM, Mattson JD, et al. Development, cytokine profile and function of human interleukin 17-producing helper T cells. Nat Immunol. 2007;8:950-7 score) and assessed variables revealed no significant positive correlation between PASI and any of the measured parameters. In the literature, there are studies confirming no correlation between IL17A, IL-22 mRNA and protein levels with PASI, and others stating the opposite.

For instance, Lowes et al. indicated a positive correlation between IL-17 and IL-22 mRNA and disease activity in the skin lesions of patients with psoriasis. ${ }^{13}$ In another study, Takahashi et al. indicated a positive correlation between the serum IL-17 levels and PASI. ${ }^{15}$ In separate studies, a significant positive correlation was also observed between the serum IL-22 concentration and PASI or disease severity. ${ }^{17-19}$

These results are contrary to the findings of de Oliveira et al. who can not show any correlation between serum levels of IL-17A and IL-22 and disease activity. ${ }^{20}$ These controversies may be related to different factors such as heterogeneity of the inclusion criteria, study population, different types of psoriasis, and different mean value of PASI in evaluated patients. ${ }^{20,21}$

\section{CONCLUSION}

The results obtained in the present study confirm again the complex nature of the immunological changes in psoriasis. These data also indicate that Th17 cells and their products may play a role in the pathogenesis of this important skin disease. For this reason, further studies should be performed regarding the precise role and mechanism of action of these cells in the initiation and promotion of disease. $\square$

13. Lowes MA, Kikuchi T, Fuentes-Duculan J, Cardinale I, Zaba LC, Haider AS, et al. Psoriasis vulgaris lesions contain discrete populations of Th1 and Th17 T cells. J Invest Dermatol. 2008;128:1207-11.

14. Roh NK, Han SH, Youn HJ, Kim YR, Lee YW, Choe YB, et al. Tissue and Serum Inflammatory Cytokine Levels in Korean Psoriasis Patients: A Comparison between Plaque and Guttate Psoriasis. Ann Dermatol. 2015;27:738-43.

15. Takahashi H, Tsuji H, Hashimoto Y, Ishida-Yamamoto A, lizuka H. Serum cytokines and growth factor levels in Japanese patients with psoriasis. Clin Exp Dermatol. 2010;35:645-9.

16. Yilmaz SB, Cicek N, Coskun M, Yegin 0, Alpsoy E. Serum and tissue levels of IL-17 in different clinical subtypes of psoriasis. Arch Dermatol Res. 2012;304:465-9.

17. Michalak-Stoma A, Bartosińska J, Kowal M, Juszkiewicz-Borowiec M, Gerkowicz A, Chodorowska G. Serum levels of selected Th17 and Th22 cytokines in psoriatic patients. Dis Markers. 2013;35:625-31.

18. Wolk K, Witte E, Wallace E, Döcke WD, Kunz S, Asadullah K,et al. IL-22 regulates the expression of genes responsible for antimicrobial defense, cellular differentiation, and mobility in keratinocytes: a potential role in psoriasis. Eur $J$ Immunol. 2006;36:1309-23.

19. Yamamoto M, Imai Y, Sakaguchi Y, Haneda T, Yamanishi K. Serum cytokines correlated with the disease severity of generalized pustular psoriasis. Dis Markers. 2013;34:153-61.

20. de Oliveira PS, Cardoso PR, Lima EV, Pereira MC, Duarte AL, Pitta Ida R, et al. IL-17A, IL-22, IL-6, and IL-21 Serum Levels in Plaque-Type Psoriasis in Brazilian Patients. Mediators Inflamm. 2015;2015:819149.

21. Kyriakou A, Patsatsi A, Vyzantiadis TA, Sotiriadis D. Serum levels of TNF- $\alpha$, IL$12 / 23 p 40$, and IL-17 in plaque psoriasis and their correlation with disease severity. J Immunol Res. 2014;2014:467541.

How to cite this article: Mansouri M, Mansouri P, Raze AA, Jadali Z. The potential role of Th17 lymphocytes in patients with psoriasis. An Bras Dermatol.2018;93(1):63-6. 